\title{
Dispositivo para monitoramento da qualidade do ar proveniente da emissão de monóxido de carbono (CO) por veículos automotores
}

\author{
André Luiz da Rocha Lima*, Valéria Loureiro da Silva
}

Faculdade SENAI CIMATEC. Av. Orlando Gomes, 1845. Piatã. Salvador-BA (CEP 41650-010). E-mail: andre.lima@al.senai.br.

Resumo. Este trabalho descreve o desenvolvimento de um dispositivo para monitoramento da emissão de gases poluentes, mais precisamente o monóxido de carbono (CO), sendo ele baixo custo e uma contribuição para a população em geral e para o meio ambiente. O dispositivo (protótipo) é baseado em técnicas de arquitetura de software e hardware open source (fontes abertas). O controle dessas informações será feito por um microcontrolador que receberá as informações das emissões de gases poluentes pelo veículo de Ciclo Otto, através de um sensor que enviará pela plataforma de comunicação (Módulo Bluetooth), para um Smartphone, e através de um aplicativo é possível verificar essas informações. Para verificação do funcionamento do dispositivo foram feitas medições no núcleo de inspeção veicular no Centro de Formação Profissional Gustavo Paiva, em Maceió-AL, Brasil. Como resultado deste projeto, verificou-se que há uma boa correlação entre os valores medidos pelo analisador de gases Discovery G4 e do dispositivo testado.

Palavras-chave: Monitoramento; Gases poluentes; Microcontrolador; Bluetooth; Monóxido de carbono.

Abstract. Device for monitoring air quality providing the emission of carbon monoxide (CO) by automotive vehicles. This work describes the development of a device to monitor the emission of pollutant gases, more precisely the carbon monoxide (CO), being the same of low cost, the same is a contribution for the population in general and for the environment. The device (prototype) is based on open source software and hardware architecture techniques. The control of this information will be done by a microcontroller that will receive information on the emission of pollutant gases by the Otto Cycle vehicle through a sensor and send them through the communication platform (Bluetooth module), to a Smartphone, and through an application is verify this information. To verify the operation of the device, measurements were taken at the vehicle inspection core at the Gustavo Paiva Professional Training Center, at Maceió-AL, Brazil. As a result of this project, it was found that exist a good correlation between the values measured by the Discovery G4 gas analyzer and the tested device.

Keywords: Monitoring; Polluting gases; Microcontroller; Bluetooth; Carbon monoxide.
Recebido:

29/10/2016

Aceito:

27/12/2016

Publicado:

$31 / 12 / 2016$

Acesso Aberto

Artigo completo

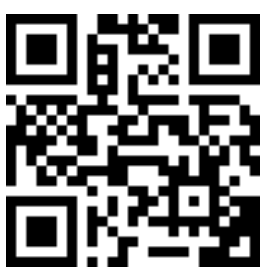

ORCIID

(D) 0000-0003-1372-9119

André Luiz da Rocha

Lima

D) 0000-0001-5466-7933

Valéria Loureiro da Silva 


\section{Introdução}

A poluição ambiental se tornou um sério problema para o ser humano, onde o ar está cada vez mais poluído e assim causando diversas doenças para a população, na qual a classe mais afetada com essa poluição são as crianças e os idosos. Segundo Oga (2008), o planeta Terra é envolvido por uma fina camada de gases que permanece em contato com a superfície do nosso planeta através da gravidade. Essa camada é conhecida como atmosfera. A autora ainda afirma que na atmosfera é jogado todos os dias uma mistura de gases tais como nitrogênio $\left(\mathrm{N}_{2}\right)$, oxigênio $\left(\mathrm{O}_{2}\right)$, argônio $(\mathrm{Ar})$, dióxido de carbono $\left(\mathrm{CO}_{2}\right)$, monóxido de carbono (CO) entre outros, constituindo assim o ar que respiramos.

Conforme Baird (2002), fontes poluidoras tais como, indústrias, queima de lixo, fornos, caldeiras e veículos automotores e outros, contribuem para o aumento da poluição atmosférica, dentre essas fontes, o crescimento da frota de veículos automotores contribuem de forma acentuada para a poluição do ar principalmente nas grandes cidades, causando problemas para a saúde e o bemestar do ser humano. De acordo com a Resolução CONAMA n ${ }^{\circ}$ 3/1990 (Brasil, 1990), que padroniza a quantidade de poluentes emitidos na atmosfera e que se ultrapassados as concentrações máximas podem afetar a saúde e o bem-estar da população, e também causar danos à flora e fauna, e aos materiais como obras públicas.

De acordo com Hasenfratz et al. (2014), hoje em dia, nos grandes centros urbanos a poluição do ar é monitorada por redes de estações de medição estáticas, onde estas estações são altamente confiáveis e capazes de medir com precisão uma ampla gama de poluentes atmosféricos, porém o seu preço de aquisição é elevado e os custos com manutenção limitam severamente o número de instalações dessas estações.

Com o desenvolvimento da eletrônica, veio os dispositivos como os sensores, plataforma de comunicação e microcontroladores, que se tornaram de baixo custo, com baixo consumo energético, e ainda pode se comunicar com os Smartphone através de aplicativos, dessa forma possibilitou o monitoramento da qualidade do ar, proposto por alguns autores como por exemplo, Meade et al. (2013) demonstrou o monitoramento da qualidade do ar utilizando uma rede densa de sensores eletroquímicos de baixo custo. Devarakonda et al. (2013), desenvolveu uma rede de sensores para monitoramento da poluição do ar em tempo real, utilizando sensoriamento móvel. Abraham e Li (2014), apresentaram um sistema de rede de sensores sem fio para aplicações de monitoramento de qualidade do ar interior. Andersen et al. (2012), propõe um sistema de sensor que visa ao monitoramento da qualidade do ar nas cidades.

Este trabalho propõe o desenvolvimento do dispositivo para monitoramento da qualidade do ar, oriundo de gases poluentes emitidos por veículos automotores, mais precisamente o monóxido de carbono (CO), o dispositivo é de baixo custo, o mesmo é uma contribuição para a população em geral e para o meio ambiente, que mostrará as informações de nível de poluição por monóxido de carbono (CO) em um Smartphone através de um aplicativo, e ainda é baseado em técnicas de arquitetura de software e hardware Open Source (fontes abertas). Além de ter como objetivo desenvolver um dispositivo para monitoramento da qualidade do ar, proveniente da emissão de monóxido de carbono (CO) pelos veículos automotores, de custo baixo em relação ao existente no mercado, utilizando plataforma microcontrolada (Arduino), plataforma de comunicação via Bluetooth e aplicativo para Smartphone.

\section{Metodologia}

Uma visão geral do protótipo é mostrada na Figura 1 e sua montagem na Figura 2. Basicamente, o funcionamento do 
sistema é iniciado a partir do momento em que o modulo sensor detecta a emissão de CO vindo através do sistema de exaustão do veículo automotor, onde envia um sinal elétrico para o Arduino, que processa esse sinal elétrico e envia outro sinal elétrico para o módulo de comunicação Bluetooth que por sua vez envia a informação através de rádio frequência para o smartphone onde disposto de um aplicativo o usuário obtém a informação da quantidade em parte por milhão (ppm) da emissão de monóxido de carbono (CO). Já na Figura 3 é mostrado o esquema elétrico do protótipo.

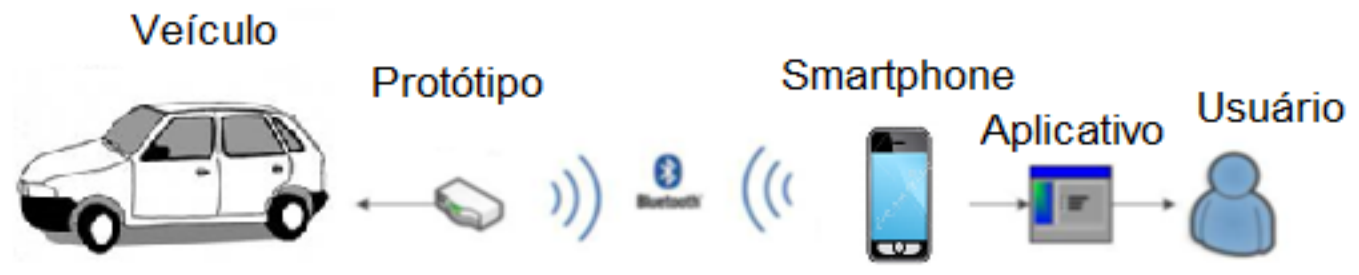

Figura 1. Visão geral do protótipo.

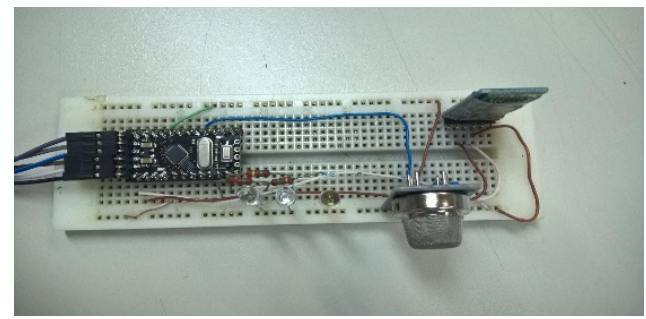

Figura 2. Protótipo montado em pront-o-board.

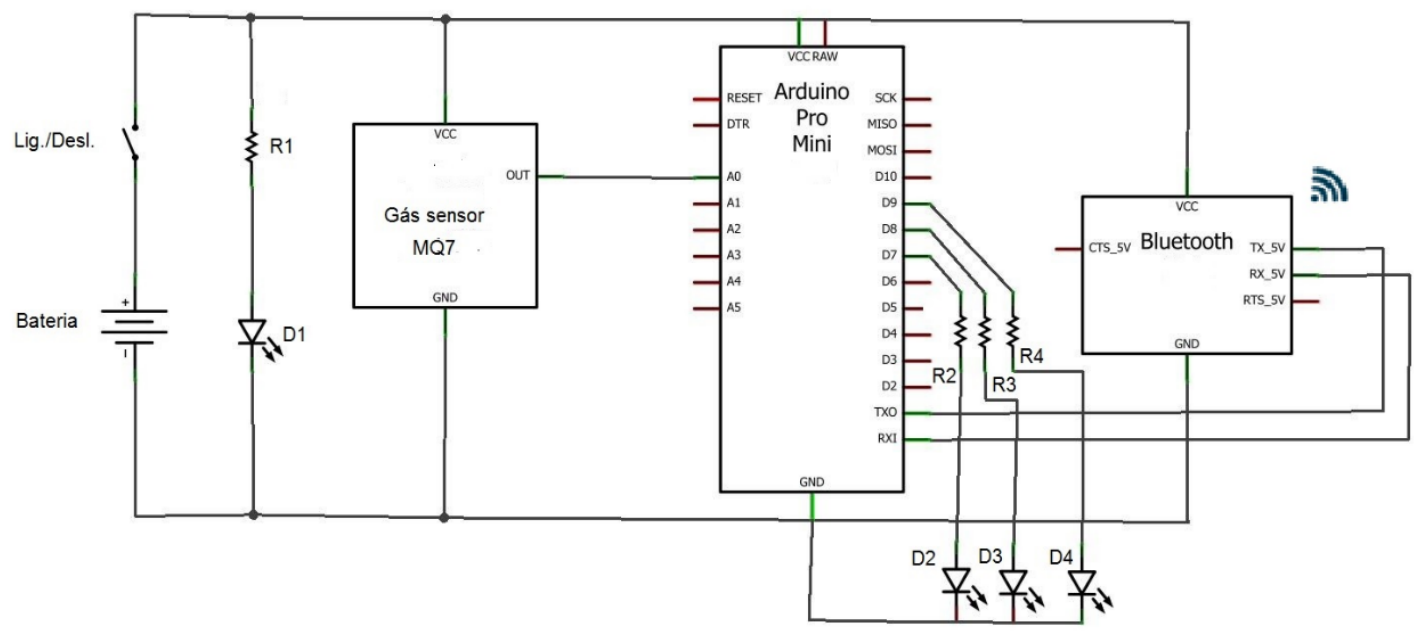

Figura 3. Esquema elétrico. 
Para o funcionamento do protótipo foi utilizado uma fonte de alimentação de 220 vac para 12 vdc, com uma corrente de $800 \mathrm{~mA}$, na saída da fonte foi acoplado um CI regulador de tensão par estabilizar a tensão em 5 vdc, pois o Arduino, o sensor MQ 7 e o módulo Bluetooth HC 05 trabalham com alimentação de 5 vdc. $\mathrm{O}$ protótipo tem um consumo de corrente em torno de $182,7 \mathrm{~mA}$, quando todos os módulos estão em pleno funcionamento. Desses 182,7 mA, 22,7 mA são consumidos pelo Arduíno, 116,8 mA pelo sensor e 43,2 mA pelo Módulo bluetooth HC 05 em estado de espera e 20,3 ma em comunicação com o smartphone.

\section{Sensor MQ 7}

Nesse protótipo foi utilizado o sensor MQ 7 como mostra a figura 4, conforme o datasheet da empresa Hanwei (2016), o MQ 7 é sensor químico composto por um microtubo de cerâmica $\left(\mathrm{Al}_{2} \mathrm{O}_{3}\right)$ e uma camada sensível de dióxido de estanho $\left(\mathrm{SnO}_{2}\right)$, apresenta menos condutividade em ar limpo. Detecta o monóxido de carbono quando está aquecido em baixa temperatura e quanto mais em contato com o gás, sua condutividade é aumentada, tem duas saídas sendo uma analógica e outra digital, na saída analógica entrega um valor de tensão analógica e na saída digital entrega valores em nível lógico 0 e 1 . Mede concentrações de monóxido de carbono na faixa de 10 a $10.000 \mathrm{ppm}$, opera entre -10 e $50^{\circ} \mathrm{C}$ e consome menos de $350 \mathrm{~mW}$ a $5 \mathrm{v}$.

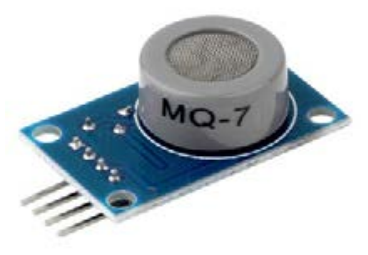

Figura 4. Módulo sensor MQ 7.

De acordo com o datasheet da empresa Hanwei (2016), para ajustar a sensibilidade do sensor MQ7, inicialmente deve-se alimentar sua resistência interna com $5 \mathrm{v}$ de tensão continua por um tempo de $60 \mathrm{~s}$ e $1,4 \mathrm{v}$ de tensão continua por um tempo de $90 \mathrm{~s}$, esse procedimento pode ser implementado por software na hora de iniciar o funcionamento do sensor. A Figura 5, mostra o sensor em blocos, sendo um para o aquecimento do mesmo que é representado pelo bloco $\mathrm{VH}$, um para a alimentação do circuito que é representado pelo bloco VC e outro é a resistência para ajustar a sensibilidade do sensor que é representado por um resistor de $10 \mathrm{k} \Omega$.

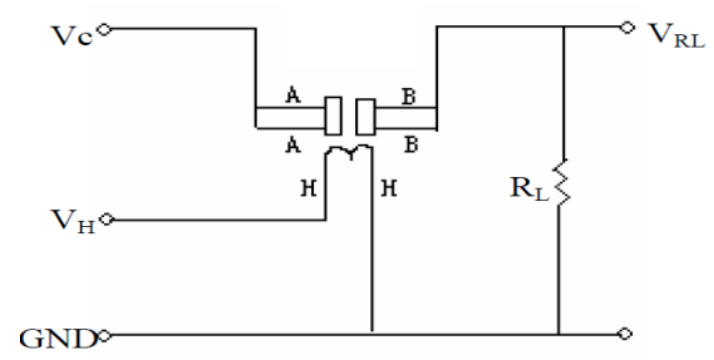

Figura 5. Esquemático do sensor MQ-7.

Conforme datasheet da empresa Hanwei (2016), Para o sensor MQ 7 funcionar corretamente deve-se aquecer os componentes sensíveis, assim deve ser conectado $5 \mathrm{v}$ nos pinos do aquecedor do sensor, no caso os pinos $\mathrm{H}$, como mostra a Figura 6, para que o mesmo chegue a temperatura de funcionamento. Ao colocar tensão nos pinos A, faz com que o sensor envie um valor analógico de tensão para os pinos B. Quando colocado uma resistência entre a saída e o GND do sensor, determina-se o quanto é sensível o sensor.
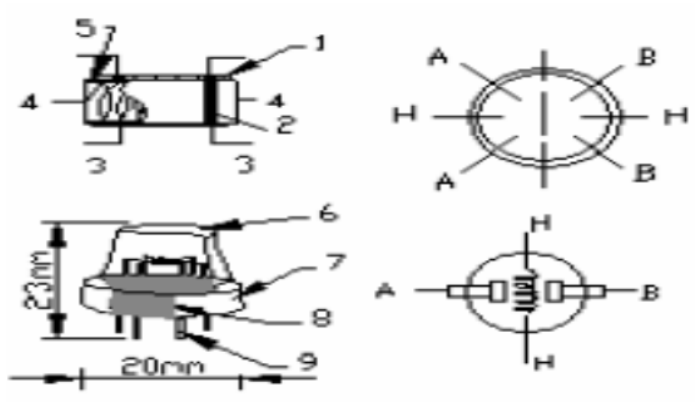

Figura 6. Os pinos do sensor CO. 
Em conformidade com datasheet da empresa Hanwei (2016), as características de sensibilidade do sensor MQ 7 para alguns gases a uma temperatura de $20{ }^{\circ} \mathrm{C}$ e usando um resistor RL de $10 \mathrm{k} \Omega$ é mostrada na Figura 7, onde R0 é a resistência do sensor em 100 partes por milhão (ppm) de monóxido de carbono e Rs é a resistência do sensor. No eixo $\mathrm{X}$ temos as concentrações em partes por milhão (ppm) e no eixo $\mathrm{Y}$ os valores de Rs/R0.

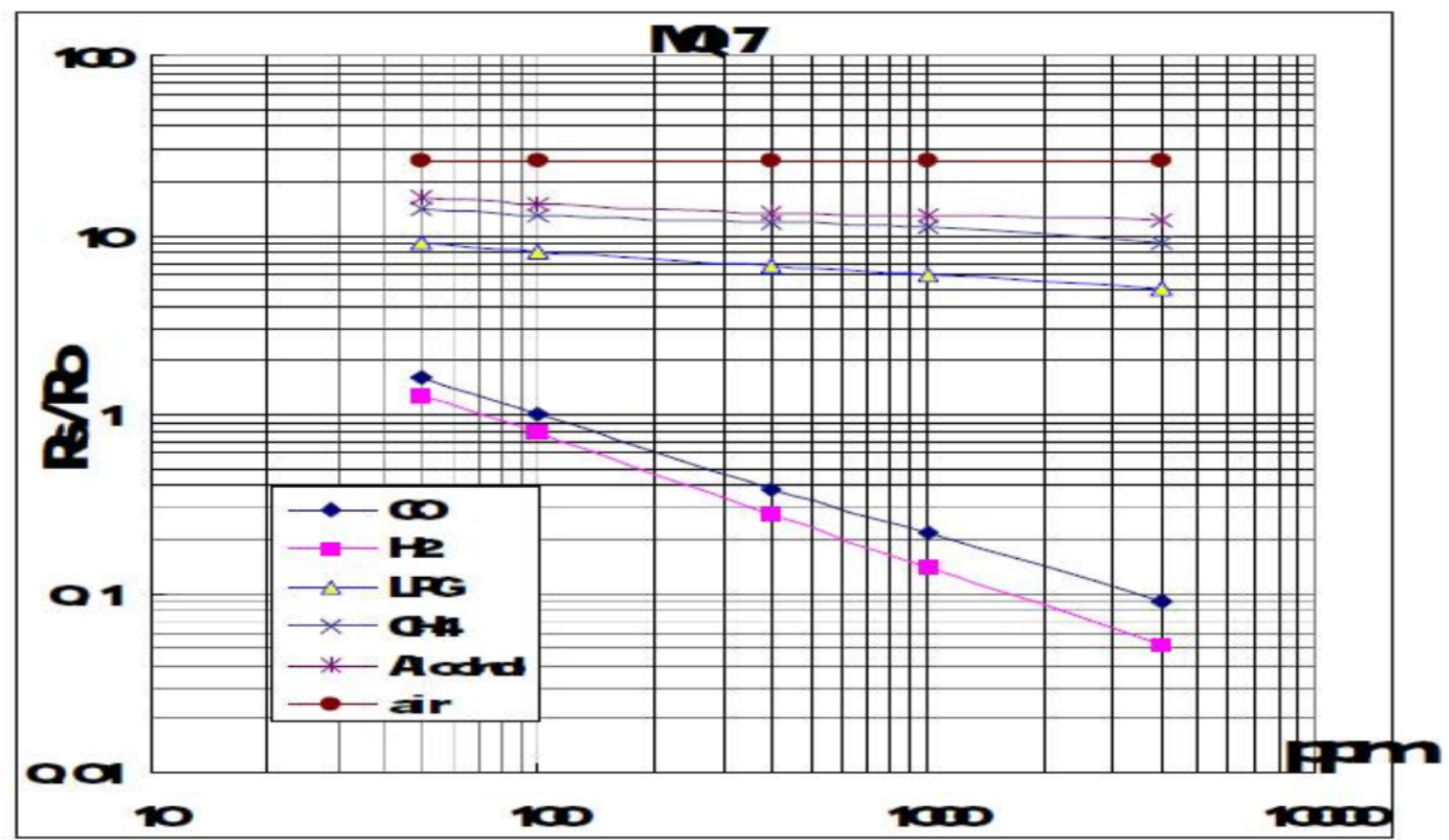

Figura 7. Característica sensitiva do sensor MQ 7.

\section{Módulo Bluetooth}

De acordo com datasheet da empresa Itead (2016), o módulo bluetooth é utilizado para fazer a comunicação de pequenos dispositivos eletroeletrônicos, como smartphone, notebook, scanners, PDAs impressoras, mouse sem fio e até mesmo qualquer dispositivo que utiliza um chip bluetooth.

Conforme datasheet da empresa Itead (2016), o uso do Módulo Bluetooth tem como objetivo principal transmitir os dados sem a necessidade da conexão física, ou seja, as ligações de cabos, entre os dispositivos eletroeletrônicos, operam na faixa de 2,4 GHz de frequência, de acordo ao seu alcance pode variar entre $10 \mathrm{a}$ $100 \mathrm{~m}$, e sua velocidade de transferência de dados melhorada (EDR) é da ordem de 3 Mbps (megabits por segundo).
Na Figura 8, é possível observar o módulo Bluetooth HC 05, o qual é utilizada neste dispositivo (Protótipo).

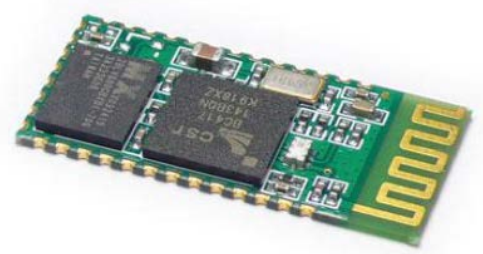

Figura 8. Módulo Bluetooth HC 05.

Em conformidade com o datasheet da empresa Itead (2016), a seguir são 
listadas algumas características do Módulo Bluetooth HC 05:

- Protocolo Bluetooth: v2.0+EDR;

- Firmware: Linvor 1.8;

- Frequência: 2,4 GHz Banda ISM;

- Modulação: GFSK;

- Emissão de energia: $<=4 \mathrm{dBm}$, Classe 2;

- Sensibilidade: $<=84 \mathrm{dBm}$ com $0,1 \%$ BER;

- Velocidade Assíncrono: 2,1 Mbps (Máx)/160 Kbps;

- Velocidade Síncrono: 1 Mbps/1 Mbps;

- Segurança: Autenticação e Encriptação;

- Perfil: Porta Serial Bluetooth;

- Suporta modo Escravo (Slave) e Mestre (Master);

- $\quad$ CSR chip: Bluetooth v2.0;

- Tensão de alimentação: 3,3 v-5 v;

- Corrente: Pareado $35 \mathrm{~mA}$; conectado 8 $\mathrm{mA}$;

- Temperatura: $-40 \sim+105{ }^{\circ} \mathrm{C}$;

- Alcance: $10 \mathrm{~m}$;

- $\quad$ Baud Rate:4800; 9600; 19200; 38400; 57600; 115200; 230400; 460800; 921600; 1382400;

- Dimensões: 26,9 x 13 x 2,2mm;

- Peso: 9,6 g.

\section{Arduino}

Consoante com Lemos (2013), o arduino é uma ferramenta de hardware livre, que proporciona a integração entre hardware e software, projetado em placa única contendo pinos de entrada e saída para a comunicação com os dispositivos externos e utiliza um ambiente de desenvolvimento, onde a linguagem de programação usada é a $\mathrm{C} / \mathrm{C}++$.

Na Figura 9, é possível observar a placa Arduino Pro Mini, a qual é utilizada neste dispositivo (Protótipo).

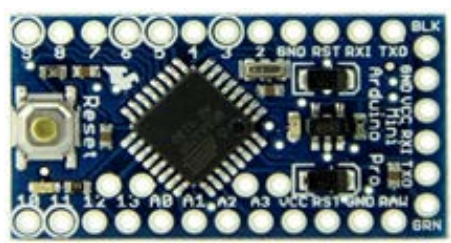

Figura 9. Arduino Pro Mini.
A seguir são listadas algumas características do Arduino Pro mini:

- Microcontrolador: ATmega328p;

- Tensão de Operação: 5v;

- Tensão de Entrada: 5-12v;

- Entradas e saídas digitais: 14 (das quais 6 podem ser usadas como saídas PWM);

- Entradas analógicas: 8;

- Corrente DC de operação: 40 mA;

- Memória flash: $32 \mathrm{~KB}$;

- SRAM: $1 \mathrm{~KB}$;

- EEPROM: $1 \mathrm{~KB}$;

- Velocidade de clock: $16 \mathrm{MHz}$;

- Dimensões: 33 x 18 x 6mm.

\section{Aplicativo}

$\mathrm{O}$ aplicativo utilizado nesse projeto foi o BT Terminal com versão 1.0.1 free, usado num smartphone com sistema operacional Windows phone 8.1, como mostra a figura 10 , já as figuras 11 e 12 , mostra na sequência a tela onde aparece a lista de dispositivos (Devices) para a conexão e logo após a tela de comunicação, ou seja, resultados.

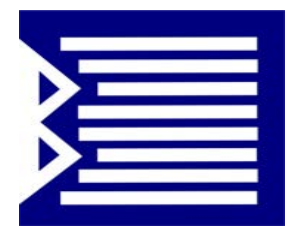

Figura 10. Ícone do aplicativo.

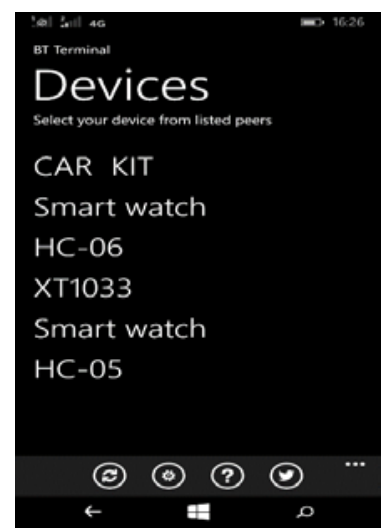

Figura 11. Lista de dispositivos. 


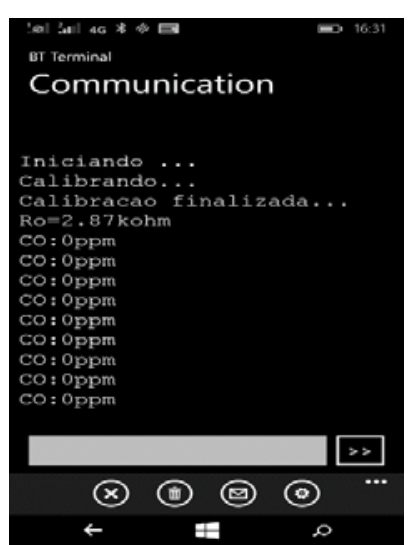

Figura 12. Tela de comunicação.

A coleta dos dados do protótipo deu-se no núcleo de inspeção veicular (NAIV), no Centro de Formação Profissional Gustavo Paiva (CFP-GP) no município de Maceió. Os dados foram comparados com os do analisador de gases Discovery G4 fabricado pela Alfatest, que é usado pelo próprio núcleo de inspeção veicular (NAIV) para avaliar a emissão de gases inclusive de monóxido de carbono (CO) dos veículos automotor e operando em marcha lenta com um tempo de exposição de dois minutos para cada medição de combustível, conforme Brasil (2009).

Para coletar os dados é feito uma inserção da sonda do analisador de gases Discovery G4 na parte interna do sistema de exaustão do veículo automotor, ou seja, dentro do escape do veículo automotor, já usando o protótipo, a coleta dos dados é feita na parte externa do sistema de exaustão do veículo automotor, ou seja, fora do escape do veículo automotor, como mostra Figuras 13 e 14.

A Tabela 1 mostra uma comparação entre as especificações do módulo sensor MQ 7 testado e do analisador de gases Discovery G4 usado como referência.

Conforme a Brasil (2009), que trata dos limites máximos de emissão de CO por veículos automotores, traz a Tabela 2 como referência para esses limites.

A Tabela 3 mostra as características principais e condições de testes para os veículos testados. Note que a escolha dos veículos com fabricação entre 1995 e 2014 permite explorar uma faixa de emissão de CO e $<0,3 \%$ a possivelmente $>3,0 \%$ se 0 veículo mais antigo estiver fora máximo do limite regulatório.

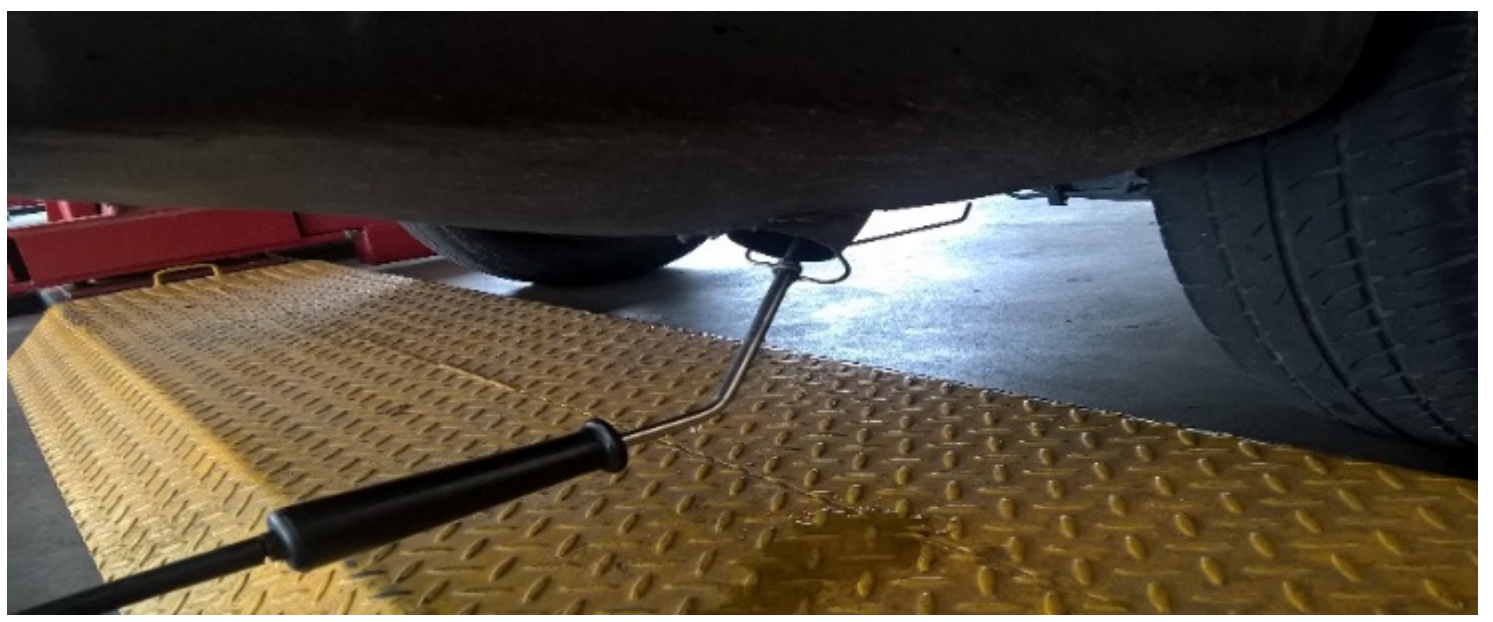

Figura 13. Sonda do Discovery 4. 


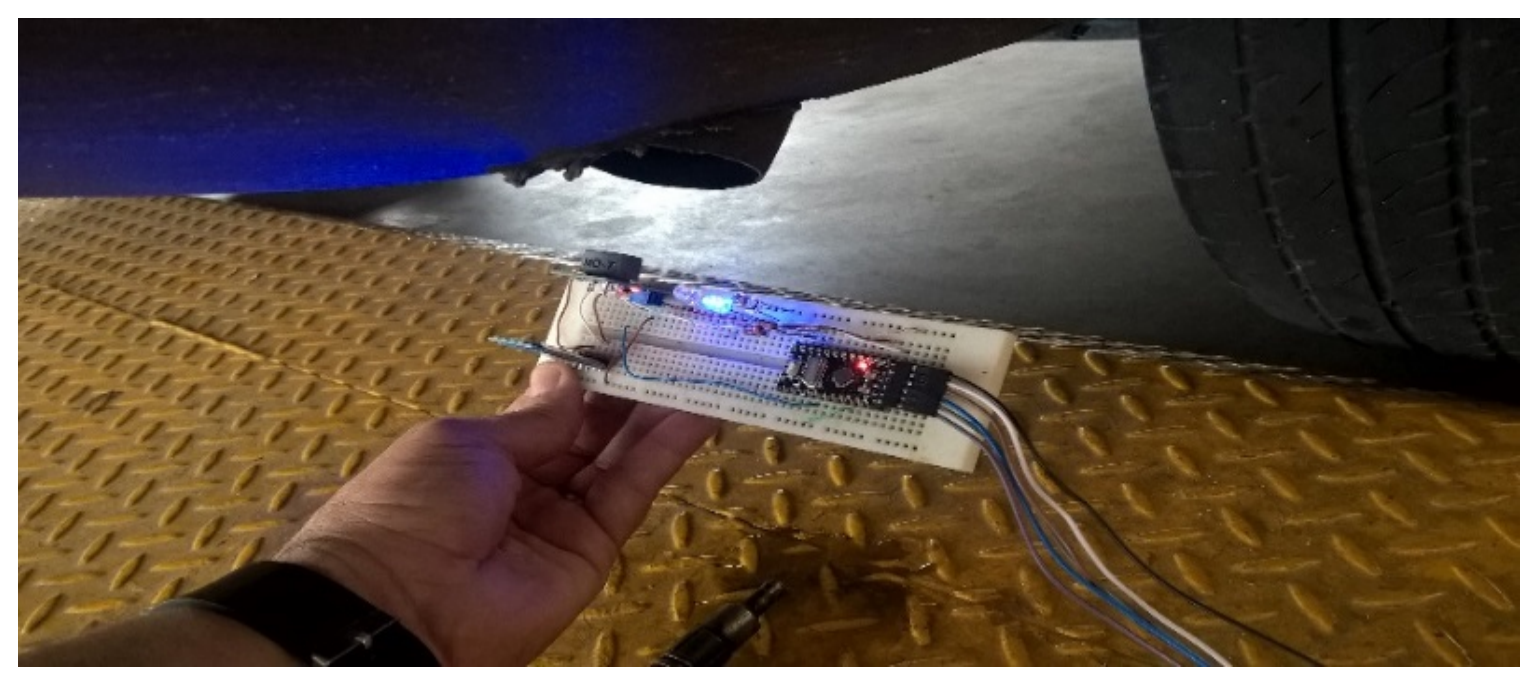

Figura 14. Protótipo.

Tabela 1. Faixa de medição do módulo sensor MQ 7 e o Discovery G4.

\begin{tabular}{ccc}
\hline Gases Medidos & $\begin{array}{c}\text { Módulo MQ 7 } \\
\text { Faixa de medição }\end{array}$ & $\begin{array}{c}\text { Discovery G4 } \\
\text { Faixa de medição }\end{array}$ \\
\hline $\mathrm{HC}$ & & $2000 \mathrm{ppm}$ vol. (Hexano) \\
& --------- & 4000 ppm vol. (Propano) \\
$\mathrm{CO}_{2}$ & ------- & $20 \%$ vol. \\
$\mathrm{CO}$ & $10 \mathrm{a} 10000 \mathrm{ppm}$ & $15 \% \mathrm{vol}$. \\
$\mathrm{O}_{2}$ & ---------- & $25 \% \mathrm{vol}$. \\
$\mathrm{NO}_{2}$ & --------- & $5000 \mathrm{ppm}$ vol. \\
\hline
\end{tabular}

Tabela 2. Limites máximos de emissão de CO, em marcha lenta e a 2500 rpm.

\begin{tabular}{|l|l|l|l|l|}
\hline \multirow{2}{*}{ Ano de fabricação } & \multicolumn{4}{|l|}{ Limites de CÓlido (\%) } \\
\cline { 2 - 5 } & Gasolina & Álcool & Flex & Gás Natural \\
\hline Todos até 1979; & 6,0 & 6,0 & - & 6,0 \\
\hline $1980-1988$ & 5,0 & 5,0 & - & 5,0 \\
\hline 1989 & 4,0 & 4,0 & - & 4,0 \\
\hline 1990 e 1991 & 3,5 & 3,5 & - & 3,5 \\
\hline $1992-1996$ & 3,0 & 3,0 & - & 3,0 \\
\hline $1997-2002$ & 1,0 & 1,0 & - & 1,0 \\
\hline 2003 a 2005 & 0,5 & 0,5 & 0,5 & 1,0 \\
\hline 2006 em diante & 0,3 & 0,5 & 0,3 & 1,0 \\
\hline
\end{tabular}

Tabela 3. Dados dos veículos usados para os testes.

\begin{tabular}{lllll}
\hline Montadora & Modelo & Ano de fabricação & Combustível & Combustível testado \\
\hline FIAT & UNO & 1995 & GNV/Gasolina & GNV/Gasolina \\
GM & SPIN & 2014 & GNV/Flex & GNV/Gasolina \\
GM & ASTRA & 2008 & GNV/Flex & GNV/Álcool \\
FIAT & SIENA - Tetrafuel & 2014 & GNV/Flex & GNV/Álcool \\
FIAT & STARDA WORKING & 2012 & GNV/Flex & GNV/Álcool \\
\hline
\end{tabular}




\section{Resultados e discussão}

Foram realizadas medições no sistema de exaustão nos veículos automotores nas condições detalhadas na Tabela 2 e os resultados para a concentração de monóxido de carbono (CO) são mostrados nos gráficos da Figura 15.

Conforme mostrado nos gráficos da Figura 16, existe uma boa correspondência entre os valores medidos pelo protótipo usando o sensor MQ-7 e o analisador de gases Discovery G4. Essa correspondência pode ser vista melhor na Figura 16a e 16b onde foram plotadas as respostas do protótipo versus a resposta do analisador de gases para os casos medidos. Esse gráfico mostra também a linha de tendência linear para toda a faixa de medição, com inclinação de 0,985 e correlação de 99,99\%. A diferença entre a resposta do sensor e o analisador de gases é muito pequena na faixa de concentração de CO medida.

Para os veículos ASTRA de ano 2008, SIENA TETRAFUEL de ano 2014 e a STRADA WORKING de ano 2012, não foi plotado os gráficos de respostas, por que tanto o analisador de gases Discovery G4 e protótipo (Sensor MQ 7) mediram 0\% de emissão de monóxido de carbono (CO) para os combustíveis gás natural (GNV) e álcool.
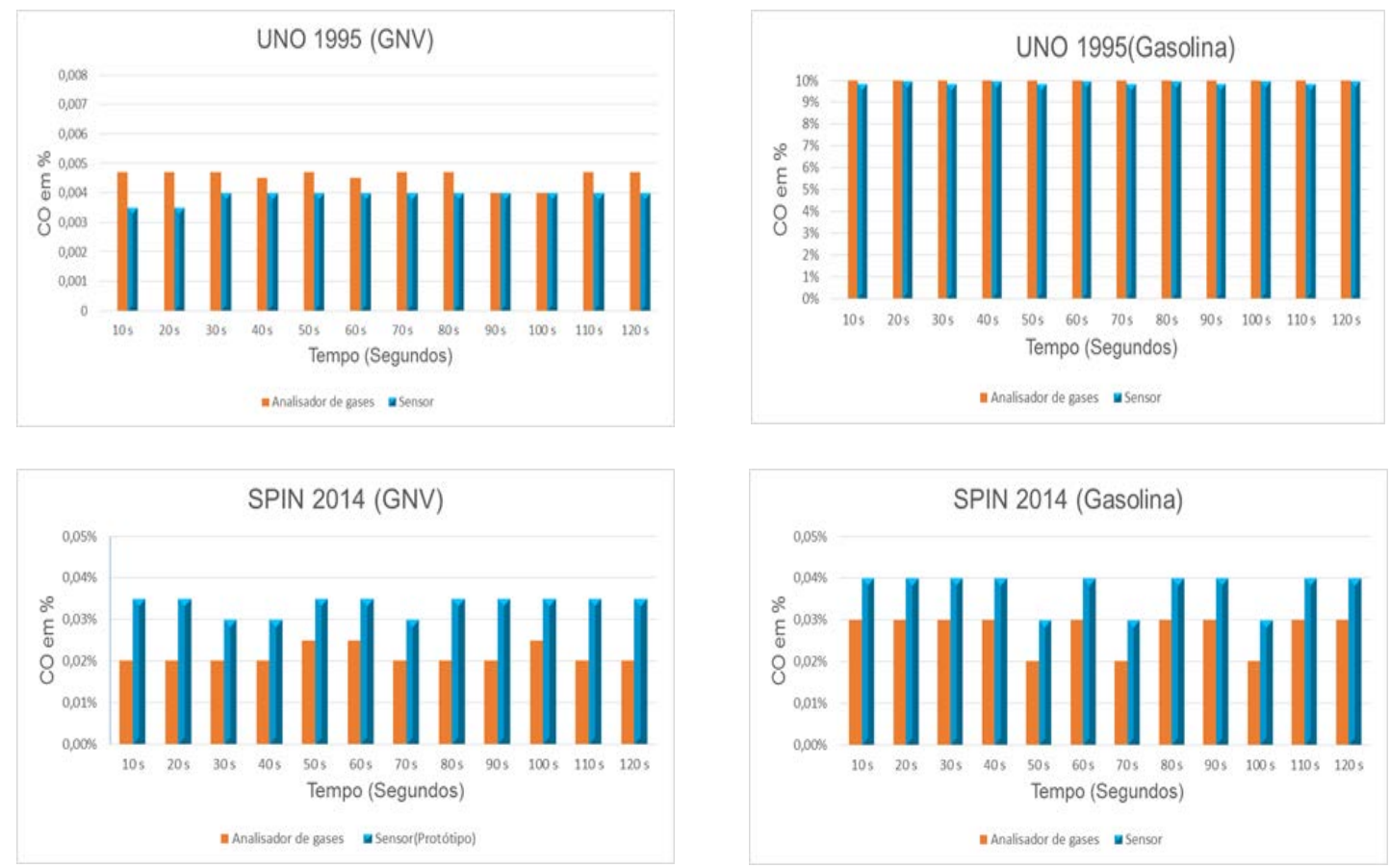

Figura 15. Gráficos da concentração de CO em \% medida para os veículos testados. 

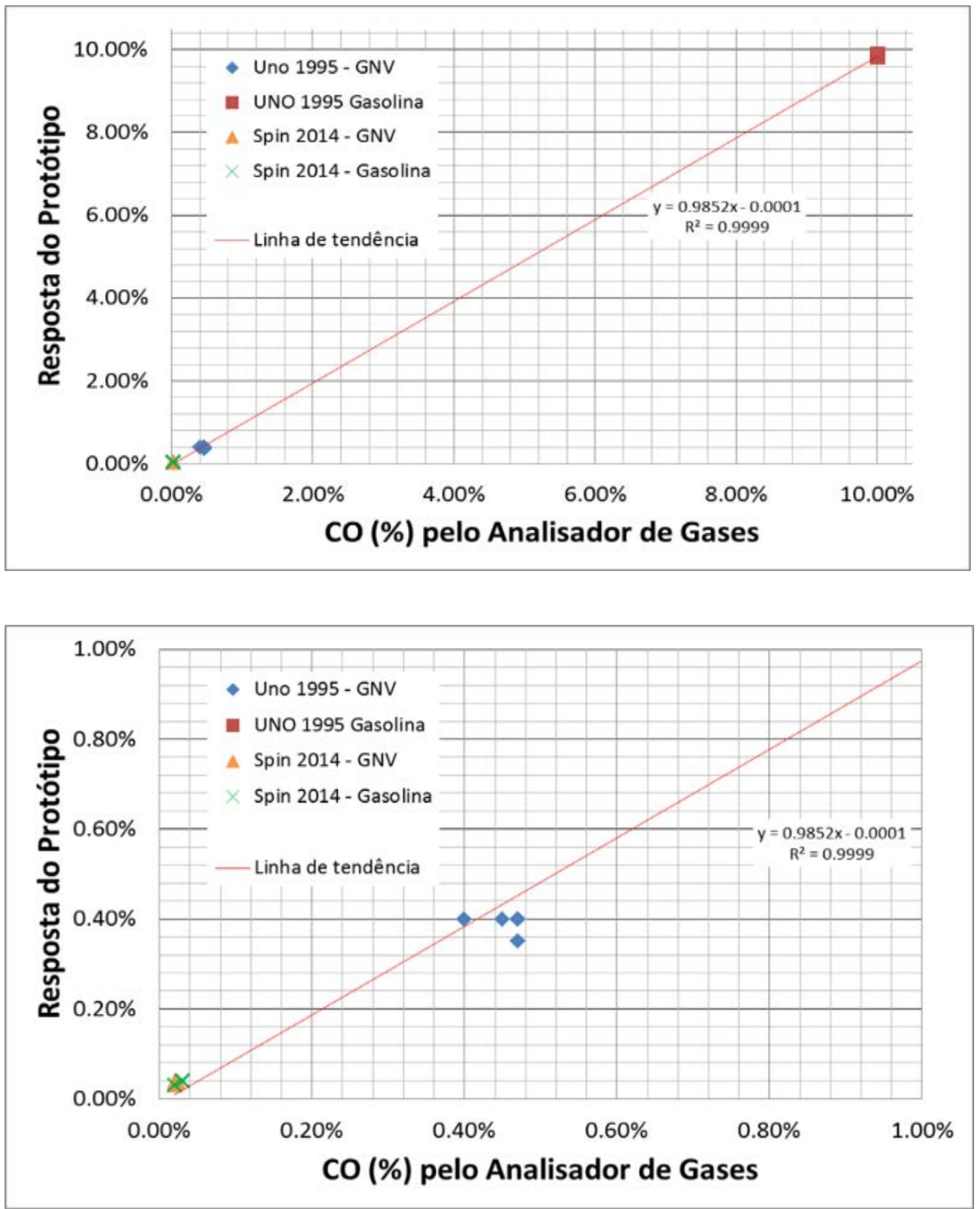

Figura 16. a) Resposta do protótipo testado em função da concentração de CO medida pelo analisador de gases Discovery G4. b) Mesmo que (a), com escala expandida para pequenos valores de $\mathrm{CO}(\%)$. 


\section{Conclusão}

Os resultados obtidos mostram uma boa correlação entre os valores de medição do analisador de gases Discovery G4 e o dispositivo desenvolvido para o monitoramento de emissão de monóxido de carbono (Protótipo).

O dispositivo (Protótipo) mencionado neste artigo apesar de simplificado servirá para visualizar as possibilidades de emprego desta tecnologia e a viabilidade técnica de implementação de sensores de baixo custo para o monitoramento da emissão de monóxido de carbono (CO) pelos veículos automotores.

Esse dispositivo (Protótipo) pode ser aplicado nos veículos automotores para monitorar a emissão de monóxido de carbono (CO) presente na exaustão do mesmo. O mesmo ainda ajudará o proprietário a planejar a manutenção do veículo, sendo assim com o motor bem regulado diminuirá o consumo de combustível e colaborar para a prevenção do meio ambiente.

\section{Declaração de conflito de interesses}

Os autores declaram não haver conflitos de interesses.

\section{Referências}

Abraham, S.; Li, X. A cost-effective wireless sensor network system for indoor air quality monitoring applications. Procedia Computer Science, $\quad$ v. 34, p. 165-171, 2014. http://dx.doi.org/10.1016/j.procs.2014.07.090

Andersen, A. B.; Krøgholt, P.; Bierre, S.; Tabard, A. NoxDroid - a bicycle sensor system for air pollution monitoring. 2012. Disponível em: <http://docplayer.net/13461665-Noxdroida-bicycle-sensor-system-for-air-pollutionmonitoring.html>. Acesso em: 22 set. 2016.

Brasil. Resolução Conama $n^{0} 3$, de 28 de junho de 1990. Dispõe sobre a qualidade do ar, previstos no PRONAR. Disponível em: <http://www.mma.gov.br/port/conama/res/res90 /res0390.html>. Acesso em: 29 ago. 2016.
Brasil. Resolução Conama no 418, de 25 de novembro de 2009. Dispõe sobre critérios para a elaboração de Planos de Controle de Poluição Veicular - PCPV e para a implantação de Programas de Inspeção e Manutenção de Veículos em Uso - I/M pelos órgãos estaduais e municipais de meio ambiente e determina novos limites de emissão e procedimentos para a avaliação do estado de manutenção de veículos em uso. Disponível em: <http://www.mma.gov.br/port/conama/res/res09 /res41809.pdf>. Acesso em: 22 jul. 2016.

Devarakonda, S.; Sevusu, P.; Liu, H.; Liu, R.; Iftode, L.; Nath, B. Real-time air quality monitoring through mobile sensing in metropolitan areas. Proceedings of the 2nd ACM SIGKDD International Workshop on Urban Computing, Article No. 15, 2013. https://doi.org/10.1145/2505821.2505834

Hasenfratz, D.; Saukh, O.; Walser, C.; Hueglin, C.; Fierz, M.; Arn, T.; Beutel, J.; Thiele, L. Deriving high-resolution urban air pollution maps using mobile sensor nodes. Pervasive and Mobile Computing, v. 16, part. B, p. 268-285, 2015. http://dx.doi.org/10.1016/j.pmcj.2014.11.008

Lemos, M. Arduino: Conheça essa plataforma de hardware livre e suas aplicações. Fazedores. 17 nov. 2013. Disponível em: $<$ http://blog.fazedores.com/arduino-conhecaesta-plataforma-de-hardware-livree-suasaplicacoes>. Acesso em: 20 ago. 2016.

Mead, M. I.; Popoola, O. A. M.; Stewart, G. B.; Landshoff, P.; Calleja, M.; Hayes, M.; Baldovi, J. J.; McLeod, M. W.; Hodgson, T. F.; Dicks, J.; Lewis, A.; Cohen, J.; Baron, R.; Saffell, J. R.; Jones, R. L. The use of electrochemical sensors for monitoring urban air quality in low-cost, high-density networks. Atmospheric Environment, $\quad$ v. 70, p. 186-203, 2013. http://dx.doi.org/10.1016/j.atmosenv.2012.11.060

Oga, S.; Camargo, M. M. A.; Batistuzzo, J. A. O. Fundamentos de toxicologia. 3. ed. São Paulo: Atheneu, 2008. 